\title{
Absolute Baseline for Testing of Electronic Distance Meters
}

\author{
Jaroslav Braun, Filip Dvořáček, Martin Štroner \\ Czech Technical University in Prague, Faculty of Civil Engineering, Department of Special \\ Geodesy, Thákurova 7, 16629 Praha 6, Czech Republic, E-mail: jaroslav.braun@fsv.cvut.cz, \\ filip.dvoracek@fsv.cvut.cz, martin.stroner@fsv.cvut.cz
}

\begin{abstract}
The paper deals with the construction and determination of coordinates of the absolute EDMs baseline in a laboratory with 16 pillars with forced centring. Leica Absolute Tracker AT401 (standard deviation of distance measurement: $5 \mu \mathrm{m}$, standard deviation of angle measurement: $0.15 \mathrm{mgon}$ ), which is designed for very accurate industrial measurements, was used for our purpose. Lengths between the baseline points were determined with a standard deviation of $0.02 \mathrm{~mm}$. The baseline is used for determining systematic and random errors of distance meters and for accuracy of distance meters at short distances common in engineering surveying for purposes of mechanical engineering.
\end{abstract}

Key words: Absolute Tracker, EDM baseline, pillars, forced-centring plates, accurate lengths.

\section{Introduction}

All surveying instruments and their measurements suffer from some errors. To refine the measurement results, it is necessary to use procedures restricting influence of the instrument errors on the measured values or to implement numerical corrections. To determine the magnitude of the errors and standard deviations of measurements of distance meters of total stations EDM (Electronic Distance Meter) baselines are used. Calibration of distance meters at the baselines are carried out as a simple regular checks [1], further as legal metrological control of measurement or for error detection and more accurate results [2]. The EDM baselines are realized as outdoor or laboratory. Outdoor baselines are usually made up of pillars with forced centring and lengths of more than $1 \mathrm{~km}[3,4]$. Lengths on these baselines are determined with an accuracy of $0.5 \mathrm{~mm}$ - $4.0 \mathrm{~mm}$ (when new determinations $0.3 \mathrm{ppm} \cdot \mathrm{D}$ or less). Distance meters are tested directly by comparing the measured distance and the reference distance of forced centring. These baselines are used mostly for routine calibration of distance meters used in common practice. Laboratory EDM baselines are used for accurate experimental measurements [5] or for calibration of total stations used in engineering. The lengths of the baselines are $20 \mathrm{~m}-50 \mathrm{~m}$. Classical laboratory baseline consists of a rail and the interferometer (standard deviation of the measured distance by the interferometer is $1.5 \mathrm{ppm} \cdot \mathrm{D},[5])$ and is determined the difference of the relative distance measured by the interferometer and by the total station.

EDM baseline combining both types of baselines, having stabilized points with forced centring and with distances determined with extra high precision was realized in geodetic laboratory at the Faculty of Civil Engineering CTU in Prague. The 16 concrete pillars with forced centring were set up and the absolute distances between the points were determined with a standard deviation of 0.02 millimetre using Leica Absolute Tracker AT401. The baseline was built for testing of the distance meters of total stations, the actual lengths between the pillars ("true values") are compared with the measured lengths. The aim of the tests is to identify random and systematic errors of measured distances and determine possible methods of its correction for usage in engineering (indoor) measurements. 


\section{Laboratory EDM Baseline}

The baseline is located in 4 basement lab rooms in Building $\mathrm{C}$ of the Faculty of Civil Engineering CTU in Prague (Figure 1). The baseline is made up of 16 concrete pillars in a single row (Figure 2). Heights of the pillars are $0.9 \mathrm{~m}$, the size of their square heads is $0.35 \mathrm{~m} \times 0.35 \mathrm{~m}$. Neighbouring pillars are mutually spaced at a distance of $0.9 \mathrm{~m}-5.0 \mathrm{~m}$ and the total length of the baseline is $38.6 \mathrm{~m}$. In the laboratory is maintained temperature about $20^{\circ} \mathrm{C}$ all year.

In 2013, the heads of the pillars were equipped by centring plates (Figure 3), which are arranged in line with the maximum lateral deviation of $2 \mathrm{~mm}$. Each centring plate is levelled in a horizontal plane (maximum slope $0.7 \%$ ). Heights of centring plates are not the same due to the different heights of pillars; their maximum difference is $50 \mathrm{~mm}$. Centring plates are turned in cylinder of hardened aluminium. Plate diameter is $140 \mathrm{~mm}$ and in the middle of it is the clamping screw. The plates are bolted to the head of the pillars in 3 points using $40 \mathrm{~mm}$ screws with dowels. The centring plates are marked by lines to tighten the tribrachs always in the same position.

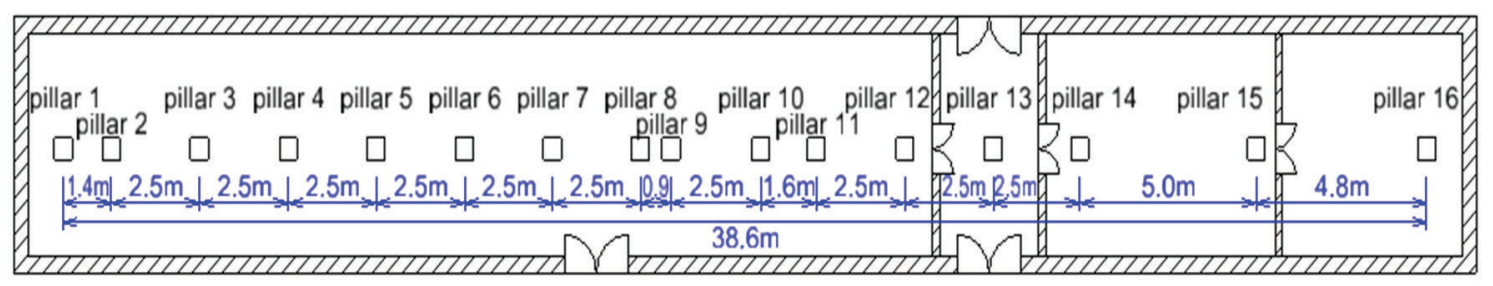

Figure 1: Scheme of the baseline
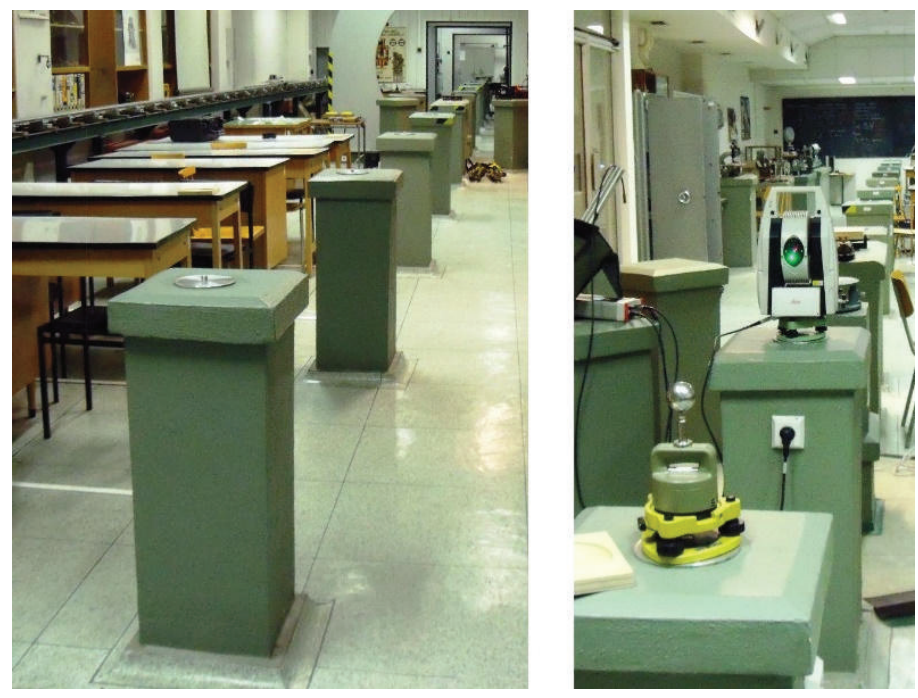

Figure 2: Photographs of the baseline

\section{Instruments and Equipment}

Leica Absolute Tracker AT401 (Figure 4) and two specially selected tribrachs of Topcon, one carrier Leica GZR3 with optical plummet and one spherical prism Leica RRR1.5 in (Figure 5) were used for determination of the size of the baseline. The Leica Absolute Tracker AT401 is primarily designed for high-precision engineering measurements. The measurement accuracy is characterized by standard deviations $\sigma_{\varphi}=\sigma_{\mathrm{z}}=0.15 \mathrm{mgon}$ for horizontal directions and zenith angles and $\sigma_{\mathrm{D}}=5 \mu \mathrm{m}$ for distances. The maximum range of the distance measurement is $160 \mathrm{~m}$. For the purpose of experimental measurements the Absolute Tracker has been borrowed from the Research Institute of Geodesy, Topography and Cartography of Czech Republic (VÚGTK). 

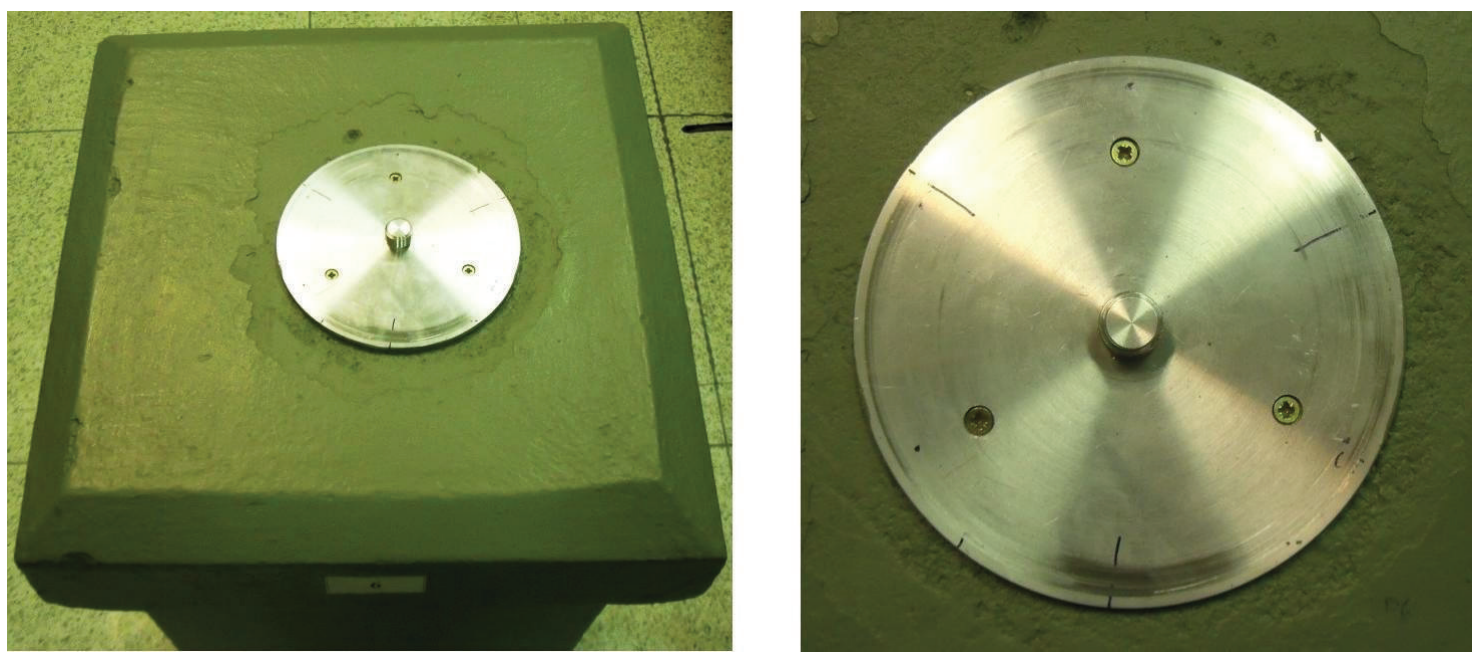

Figure 3: The head of pillar with centring plate
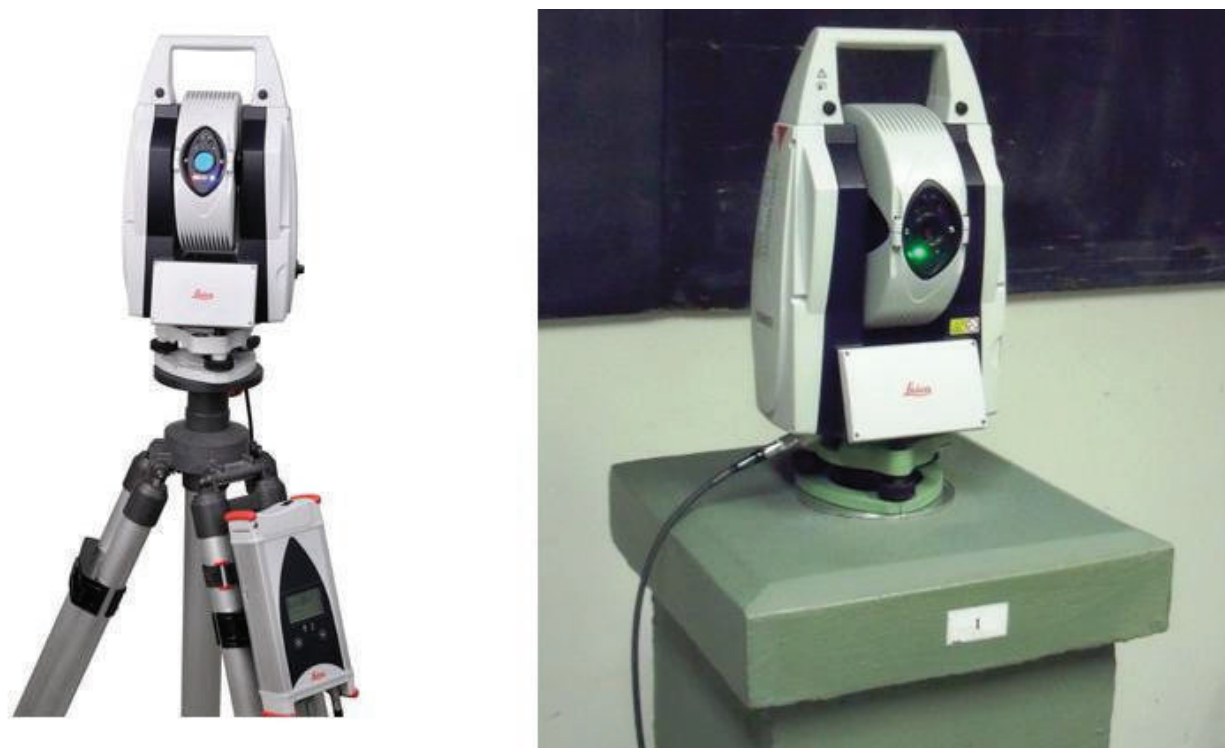

Figure 4: Leica Absolute Tracker AT401

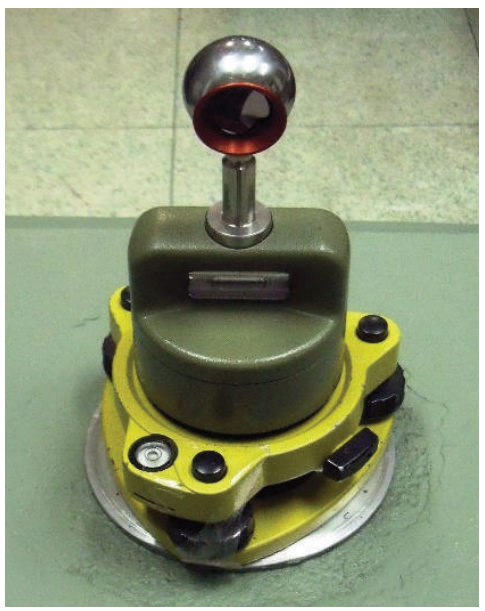

Figure 5: Target Set - Tribrach Topcon, Leica carrier GZR3, Leica prism RRR1.5 


\section{Testing of the Equipment}

It is assumed to use 2 tribrachs ( 1 for instrument and 1 for the target), 1 carrier and 1 prism for testing of the distance meters on baseline. To ensure high precision of the absolute distances determined between the pillars and the informative value of tests distance meters, it was necessary to perform a test measurement of the tools to determine their parameters so that subsequent measurements were not discarded unnecessarily because of damage.

Equipment of the facilities of the Department of Special Geodesy at Faculty of the Czech Technical University in Prague was selected to determine the baseline. Available was one precise carrier Leica GZR3 for which the manufacturer specifies the accuracy of centring at $0.3 \mathrm{~mm}$. It was also possible to use 5 Topcon tribrachs, from which two best also with similar characteristics according to the test measurement were selected and one of the three available mini prisms LeicaGMP101 was chosen. All this equipment is used for the purposes of baseline measurements only.

To verify the reliability and accuracy of tribrachs the tests of re-centring and re-attaching of the carrier and also a test of repeated rotation of the carrier in the tribrach were designed. There were also determined the addition constants of the mini prisms. All tests were performed with use of the tracker.

Appropriate carrier and tribrach together were always assembled for the test of the repeatability of centring. Screwing and levelling at the centring plate by carrier's bubble were done five times. There was maintained the same position and orientation of the tribrach screws and of the carrier. Length measurement was performed after each screwing. Between five tribrachs, the difference in length at the same pillar was up to $0.7 \mathrm{~mm}$. Sample standard deviation of repeatability of centring of individual tribrachs reached values of $0.002 \mathrm{~mm}-0.007 \mathrm{~mm}$.

For the test of repeatability of placement of carrier (verification of the functionality of locks of the tribrach), there was always screwed a tribrach on a centring plate and accurately levelled by the carrier bubble. Then the carrier was five times removed and again clamped during unchanged levelling and orientation of the carrier to the instrument. After each clamping length measurements were taken. Standard deviation of repeatability reached values of $0.002 \mathrm{~mm}-0.04$ $\mathrm{mm}$.

On the basis of these tests 2 of the tribrachs for that the test of centring showed the same position were selected and they have also a minimal standard deviation of repeated clamping of carrier to tribrach. It was taped one adjustment screw on tribrachs so that when it was re-centred, it was always equally high. Values determined in test measurements also reflect the degree of wear of tools and point to necessity of control of used equipment.

For the test of the eccentricity of the carrier tribrach with a carrier was accurately levelled. The carrier was then rotated around its axis to the 9 position and rotation was performed twice (clockwise and vice versa). After each rotation the distance was measured. There was detected approximately circular eccentricity with radius of $0.03 \mathrm{~mm}$.

Additive constants of the Leica GMP101 mini prisms were determined by the comparison of the lengths measured at the spherical prism and Leica mini prisms. Lengths were measured on three pillars of baseline and to each pillar it was measured five times. For all tested prisms additive constant of approx. $-16.5 \mathrm{~mm}$ was determined in comparison of $-16.9 \mathrm{~mm}$ specified by the manufacturer. Sample standard deviation of determined constants totals had reached values of $0.006 \mathrm{~mm}-0.03 \mathrm{~mm}$. Designated additive constant of selected mini prism is used in the testing of instruments on the baseline. 


\section{Determination of the Dimensions of the Baseline}

The aim of the measurements was to determine the horizontal distances between the pillars with accuracy better than $0.05 \mathrm{~mm}$. Measurements were taken from four standpoints and then determination of coordinates of points by the adjustment by method of least squares was made, all in July 2013.

For the measurement were chosen 3 standpoints directly on the pillars of the baseline (first and last pillar 1 and 16 and the middle pillar 10) and one eccentric standpoint (1.5 m from the axis of baseline near pillar no. 6). From each standpoint it was measured in two faces at all the pillars of baseline. To guarantee the high accuracy required, it was necessary to comply the principles of minimizing of the effects of eccentricity of equipment. The tribrachs and centring plates are marked with signs and its coincidence ensures that the tribrach is screwed always in the same position. To maintain the orientation of the carrier Leica GZR3 the eyepiece was always turned toward the first pillars. Spherical Leica prism was always rotated with Leica sign up to preserve its orientation.

\section{Calculation}

For adjustment program GNU Gama [6] was used, which allows in a simple XML file (Extensible Markup Language) to define the input file for adjustment, and allows entering various standard deviations to the individual measurements. To calculate the local coordinates XY horizontal directions and horizontal distances were used, where the standard deviation of the directions 0.3 mgon was chosen and length of $0.025 \mathrm{~mm}$, both with respect to the identified uncertainties of centring of targets.

In adjustment was considered that coordinates of the forced centring with target will be identical to the coordinates of the centre of the instrument. The adjustment, however, showed that there is a certain eccentricity, the difference in the location of device and standpoint was approx. $0.2 \mathrm{~mm}$ (due to different used tribrach). Therefore, the standpoints of the Leica Absolute Tracker on the pillars of baseline were designated as separate (free) points. In the resulting adjustment were therefore determined plane coordinates of four standpoints and sixteen pillars. The 122 measurements were used in the calculation (the horizontal directions and distances), of which 81 were redundant. In adjustment was no measured value reported to be an outlier. Mean standard deviation of the position of adjusted points reached $0.03 \mathrm{~mm}$, the maximum positional standard deviation was $0.06 \mathrm{~mm}$. A priori standard deviation was chosen 1.0 and a posteriori standard deviation after the adjustment was 0.77 . Size of a posteriori standard deviation indicates that the standard deviations of the measurements were achieved better than expected.

From adjusted coordinates were calculated horizontal distances between the baseline points, their standard deviations were $0.02 \mathrm{~mm}$ (approximately valid for all of them). These distances are now used as a reference for testing of the distance meters.

\section{Conclusion}

In geodetic laboratory of the Faculty of Civil Engineering CTU in Prague was established 38.6 meters long EDM baseline consisting of 16 concrete pillars with forced centring. Lengths between the pillars were determined using an accurate instrument Leica Absolute Tracker AT401 with use of the least square adjustment method with a standard deviation of $0.02 \mathrm{~mm}$. From time to time implementation of control is assumed - verification measurements to determine the stability of the base and constancy of used equipment.

The base is at present used for testing of distance meters instruments useful in indoor industrial measurements and for determining the size of their errors. 


\section{Acknowledgements}

The article was written with support of the internal grant of Czech Technical University in Prague SGS14 "Optimization of acquisition and processing of $3 D$ data for purpose of engineering surveying ".

\section{References}

[1] ČSN ISO 17123-4, (2005) Optics and optical instruments - Field procedures for testing geodetic and surveying instruments - Part 4: Electro-optical distance meters (EDM instruments)). Prague: Czech Standards Institute. 24 pp.

[2] RÜEGER, J. M. (1990) Electronic Distance Measurement. 3. ed. Heidelberg: SpringerVerlag, 266 pp. ISBN 3-540-51523-2.

[3] JOKELA, J., HÄKLI, P. (2006). Current Research and Development at the Nummela Standard Baseline. In: Shaping the Change - XXIII FIG Congres, Munich. 15 pp. Available:

https://www.fig.net/pub/fig2006/papers/ps05_02/ps05_02_02_jokela_hakli_0371.pdf

[4] LECHNER, J., ČERVINKA, L, UMNOV, I. (2008). Geodetic Surveying Tasks for Establishing a National Long Length Standard Baseline. In: Integrating Generations - FIG Working Week 2008. Stockholm. 9 pp.

[5] DE WUlf, A., CONSTAleS, D., MeSKens, J., NUTTENS, T., STAL, C. (2011). Procedure for Analyzing Geometrical Characteristics of an EDM Calibration Bench. In: Bridging the Gap between Cultures - FIG Working Week 2011. Marrakech. 14 pp. Available:

http://www.fig.net/pub/fig2011/papers/ts08e/ts08e_dewulf_constales_et_al_5266.pdf

[6] ČEPEK, A. (2013). GNU Gama 1.14 - Adjustment in Geodetic Networks. Edition 1.14 [online]. Available: http://www.gnu.org/software/gama/manual/index.html 